Japanese Psychological Research

Tapanese Pol. 18, No. 1, 14-19
1976, Vological Resear

\title{
CHILDREN'S SORTING BEHAVIOR AS A FUNCTION OF CONCEPTUAL ABILITY AND FREQUENCY OF INSTANCES
}

\author{
TAKESHI SUGIMURA ${ }^{1}$
}

Nara University of Education

\begin{abstract}
In order to assess S's concept utilization in learning the sorting tasks, preschool children were given either a conceptual sorting task (CST) or a half conceptual sorting task (HST). The results revealed that CST was learned faster than HST by the Ss with high conceptual ability (Exp. I) and in the sorting tasks with high frequency instances (Exp. II), and that both sortings were learned almost at the same rate by the Ss with low ability and in the sorting tasks with low frequency. The superiority of CST over HST is attributable to S's concept utilization in learning the sorting tasks. Whether preschool children can learn in a mediational manner or not depends on S's ability and the nature of the learning task.
\end{abstract}

Kendler, Kendler, and Sanders (1967) assessed the utilization of verbal mediation by the use of a procedure similar to reversal and nonreversal shifts in discrimination learning. College students were required to associate Response $\mathrm{A}$ with the instances of one conceptual category (e.g., vegetable) and Response $B$ with those of another (e.g., clothing). On the shift learning, either all instance and response associations were reversed (full reversal shift) or only half of them were reversed (half reversal shift). Kendler, et al. assumed that if $S$ s use conceptual terms as verbal mediators, full reversal shift would be learned faster than half reversal shift, and that if $S_{\text {s }}$ can not use them, reverse would be true. The result were in line with their assumption. Kendler, Kendler, and Marken (1969) studied these two types of shifts with 4-, 5-, 7-, and 18-year-old subjects.

I The author is indebted to Miss Yōko Terao for collecting the data of Experiment $\mathrm{I}$ and for editing the English manuscript, and to Miss Hiroko Ichikawa for collecting the data of Fiperiment II .

Request for reprints should be sent to Takeshi Sugimura, Nara University of Education, Takabatake-cho, Nara 630 , Japan.
The results were that the youngest $S$ s learned both shifts almost at the same rate and that full reversal shift was learned increasingly faster than half reversal shift with ages from 5 to 18 . They argued that the availability of verbal mediation increased with age, and this was consistent with the verbal mediation theory.

Although the procedure used in the above studies appears to be appropriate to assess the utilization of verbal mediation in that they employed verbal materials, there might still remain some apprehensions about their methodology. (a) Training $S$ s to respond in a conceptual manner may have differential effects on younger and older $S \mathrm{~s}$. It is rather convincing that the training may strengthen conceptual responses in older $S \mathrm{~s}$, but whether this is also true in younger $S \mathrm{~s}$ is questionable. (b) What was learned in original learning may not necessarily transfer to shift learning, especially in younger $S \mathrm{~s}$. If this is the case, it is not reasonable to infer the possibility of concept utilization from $S \mathrm{~s}^{\prime}$ performances in shift learning.

In order to eliminate these factors, Sugimura (1974) employed the following procedure which involved no shift learning. 
Half of the $S$ s were given a conceptual sorting task (CST) which required them to sort some instances of two concepts into two groups on the basis of the concepts. The other half $S$ s were given a half conceptual sorting task (HST) which required them to make a group of the half instances from each concept and another group of the remaining instances. Sugimura (1974) trained $S \mathrm{~s} 3,5,6,9$, and 20 years of age on either CST or HST with the instances of fruit and vehicle. He obtained the results that the youngest children learned CST and HST with no performance difference and that CST was learned increasingly faster than HST through ages from 5 to 20 . These findings were interpreted as evidence that $S$ 's ability to take advantage of conceptual terms as mediators increased with age.

it has been well confirmed the availability of verbal mediation (concept) is an increasing function of age. Even when children at the same age served as the $S$, however, the utilization of concepts in learning the sorting tasks would depend on some other factors. Among them $S$ 's ability and the nature of learning materials were concerned in the present study. Two separate experiments were designed to examine the effects of $S$ 's conceptual ability (Experiment I) and frequency of instances used in the tasks (Experiment II) upon learning CST and HST.

\section{EXPERIMENT I}

\section{Method}

Subjects. The $S \mathrm{~s}$ were 80 nursery school children. Their ages ranged from 5:6 to $6: 7$ with a mean of $6: 1$. They were given the conceptual ability tests. Based on their scores of these tests, $20 \mathrm{~S}$ s were selected from the upper end of the distribution and $20 \mathrm{~S}$ s from the lower end. Half of the $S$ s with higher and lower ability were given CST and the remaining $S$ s were given HST.

Materials and apparatus. The conceptual ability test was composed of two subtests. The instances used in the two subtests were familiar to children of this age, and were easy to show in pictures.

(a) Abstraction test. The abstraction test was designed to assess $S$ 's ability to abstract a common concept from its instances. $S$ was required to select one of three instances which he thought to be of the same kind as the sample instance, and to verbalize the conceptual term common to the two instances. This test was constructed after the ones used by Hata (1973) and Sugimura (1969). Eight sets of 4 colored pictures were provided for this test, and each set was mounted on a $19.0 \times 26.5 \mathrm{~cm}$ white card. One of the instances on each set was the sample instance and was mounted on the upper center of the card. The other three instances were the choice alternatives and were mounted on the bottom half of the card. Four out of 8 sets were the fruit sets and they involved an instance of fruit as the sample and instances of fruit, vegetable, and flower as the choice alternatives. One of the fruit sets, for cxample, involved a peach as the sample and a banana, a Cihinese cabbage, and a tulip as choice alternatives. The remaining 4 sets were the vegetable sets and they involved an instance of vegetable as the sample and instances of vegetable, fruit, and flower as the choice alternatives. One of the vegetable sets, for example, involved a Chinese cabbage as the sample and a tomato, a melon, and a lily as the choice alternatives. An additional card with a blue triangle as the sample and a green triangle, a red square, and a yellow circle as the choice alternatives was used for practice.

(b) Identification test. The identification test was designed to assess $S$ 's ability to identify whether the instances were of a given concept or not. $S$ was given several instances of the three concepts one by one and required to respond with "Yes" or "No" according to whether the instance belonged to a given concept or not. This test was constructed after the ones used by Neimark (1974) and Saltz, Soller, and Siegel (1972). Two lists of 8 instances were provided for this test. Each instance was a colored picture and was mounted 
on a $5.0 \times 6.5 \mathrm{~cm}$ white card. The fruit list involved 4 instances of fruit and 4 instances of other concepts ( 2 vegetables and 2 flowers). The vegetable list involved 4 instances of vegetables and 4 instances of other concepts ( 2 fruits and 2 flowers).

(c) Sorting task. The instances used in the sorting tasks were grapes and a strawberry as fruits, and a cucumber and a carrot as vegetables. These instances were selected from the upper five instances in frequency (Sugimura \& Ichikawa, 1975), and were not used in the conceptual ability test. They were colored pictures and each of them was mounted on a $5.0 \times 6.5 \mathrm{~cm}$ white card. A pack of 40 cards consisted of 10 cards of each instance was provided and was arranged in a random order with the restriction that the same instance did not appear successively. Some red circles and triangles were employed for practice trials.

A red $27 \times 15 \times 15 \mathrm{~cm}$ sorting box was used. The box possessed a slope which was made from the upper to the front side of it. Two $1.5 \times$ $8.0 \mathrm{~cm}$ openings were located side by side on this slope (see Sugimura, 1974).

Procedure. $S$ was run individually in a room of his school. He was given the conceptual ability test and then either CST or HST.

(a) Abstraction test. When $S$ was brought to the room and seated, he was presented a card with four geometric figures for practice of the abstraction test and was given the instruction, "Let's play a matching game. You can see figures on this card, can't you? The upper one is a triangle and your task is to select the one which you think to be of the same kind as this. Which one of these lower three is similar to the upper one?"

When $S$ selected the triangle from the choice alternatives, $E$ asked $S$ the reason for his choice and required to verbalize the superordinate concept common to the two figures--." Triangle." If $S$ could not select or verbalize correctly, $E$ taught $S$ the correct response and let $S$ repeat it after $E$. Following this practice, $S$ was given 8 sets one by one at $S$ 's response pace. Fruit and vegetable sets were mixed into one series and were presented in a predetermined order. $S$ 's correct choices and verbalizations were scored. The maximum score of this test was $16 ; 8$ for the correct choices and 8 for the correct verbalizations.

(b) Identification test. Following the abstraction test, $S$ was given the identification test with the instruction, "Now I will show you many pictures one by one. Tell me whether it is a kind of fruit or not." The frst instance of the fruit list was presented. $E$ said to $S$, "Is this a fruit?" and required him to answer "Yes" or "No." $E$ repeated this question for every instance. The vegetable list was given in a similar way. $E$ said to $S$, "Is this a vegetable?" for every instance. $S$ 's correct identifications were scored. That is, S's "Yes" to the instances of fruit or vegetable and "No" to the instances of the other concepts were defined as correct. Each of the two lists was consisted of 8 instances, and the maximum score of this test was also 16.

(c) Sorting task. The maximum total score of the abstraction and the identification tests was 32 , and the summed scores for all $S$ s ranged from 11 to 32 with a mean of 22.5. Based on their scores, $20 \mathrm{Ss}$ were selected from the upper end of the distribution and $20 \mathrm{~S}$ s from the lower end. The mean scores of higher and lower $S$ s were 29.1 (29-32) and 16.3 (11-18), respectively. The sorting tasks were given to these $40 \mathrm{Ss}$. $S$ was handed a card for practice and was given the instruction, "Let's play a card game. I will give you such cards as these one by one. Your task is to guess which opening of this post your card should be thrown into and to post it into the correct opening. Now you have a card with a triangle. Show me which is the correct opening for a triangle."

$S$ was given some practice trials. $E$ said "Right" for $S$ 's correct responses and "Wrong" for his errors. After making sure that $S$ understood how to perform his task, $E$ introduccd CST or HST. The cards were given with $E$ 's namings of the instances, one by one at $S$ 's pace. $S$ was trained to reach a criterion of eight successive correct responses or a maximum of 40 trials. The instances to be thrown into the right and the left openings for CST and HST are shown in Table 1. 
TABLE 1

Sorting tasks used in Experiment I

\begin{tabular}{l|l|l}
\hline & Left opening & Right opening \\
\hline \multirow{2}{*}{ CST } & Carrot & Strawberry \\
& Cucumber & Grapes \\
\hline \multirow{2}{*}{ HST } & Carrot & $\begin{array}{l}\text { Strawberry } \\
\text { Cucumber }\end{array}$ \\
\cline { 2 - 2 }
\end{tabular}

Note: CST required $S$ to throw the instances into the left and the right openings on the basis of the concepts. HST required $S$ to throw the half instances from each concept into the left opening and the other instances into the right opening.

\section{Results}

The mean numbers of trials to criterion are shown in the left half of Table 2. Four $S$ s could not reach the criterion within 40 trials, and they were given a score of 40 points. A $2 \times 2$ analysis of variance showed the significant main effect of task at the $1 \%$ level with $F(1,36)=10.29$ and the significant interaction between task and ability at the $1 \%$ level with $F(1,36)=$ 11.71. The significant interaction was due to the fact that the $S$ s with higher conceptual ability learned CST significantly faster than HST $(t=4.96, d f=36, p<.01)$, and that the $S$ s with lower conceptual ability learned both sorting tasks almost at the same rate $(t=0.15)$.

\section{EXPERIMENT II}

\section{Method}

Subjects. The Ss were 80 nursery school children of 40 boys and 40 girls. Their ages ranged from $4: 3$ to $5: 7$ with a mean of $5: 2$. They were divided into the following four groups on the basis of their scores in "Information " test of WPPSI ; high-frequency instanceCST (H-CST), high-frequency instance-HST (H-HST), low-frequency instance-CST (LCST), and low-frequency instance-HST (LHST) groups. The mean scores of Information test were $8.2,8.2,8.1$, and 8.1 , respectively, in the above order of groups.
TABLE 2

Mean number of trials to criterion in sorting tasks

\begin{tabular}{l|cc|cc}
\hline & \multicolumn{2}{|c|}{$\begin{array}{c}\text { Experiment I } \\
\text { Conceptual ability }\end{array}$} & \multicolumn{2}{c}{ Experiment II } \\
& Instance frequency \\
\cline { 2 - 5 } & High & Low & High & Low \\
\hline CST & 8.4 & 28.7 & 20.4 & 43.4 \\
HST & 33.1 & 27.9 & 32.6 & 42.9
\end{tabular}

Materials and apparatus. Each two high- and low-frequency instances of fruit and vegetable were selected from the conceptual category norms for 180 children of 3,4 , and 5 years of age (Sugimura \& Ichikawa, 1975). These norms were constructed on the basis of children's response instances to several category names. The high-frequency instances were selected from the upper five instances in frequency and the low-frequency ones were selected from the instances which occurred only one time. The instances used in this experiment are shown in Table 3. These instances were colored pictures and each of them was put on a $5.0 \times 6.5 \mathrm{~cm}$ white card. A pack of 60 cards consisting of 15 cards of each instance was provided for the high- and the low-frequency instances, respectively, and they were arranged in a random order with the restriction that the same instance did not appear successively. The cards for practice and the sorting box were the same as those used in Experiment I.

Procedure. $S$ was run individually in a room of his school. After the administration of Information test, $S$ was given the practice and CST or HST by the same procedure as in Experiment I. $S$ was trained to reach a criterion of eight successive correct responses or a maximum of 60 trials. The instances to be thrown into the right and the left openings for CST and HST are shown in Table 3.

\section{Results}

The mean numbers of trials to criterion are shown in the right half of Table 2 . The numbers of $S$ s who could not reach the criterion within 60 trials were $0,3,9$, and 7 for H-CST, H-HST, L-CST, and L- 
TABLE 3

Sorting tasks used in Experiment II

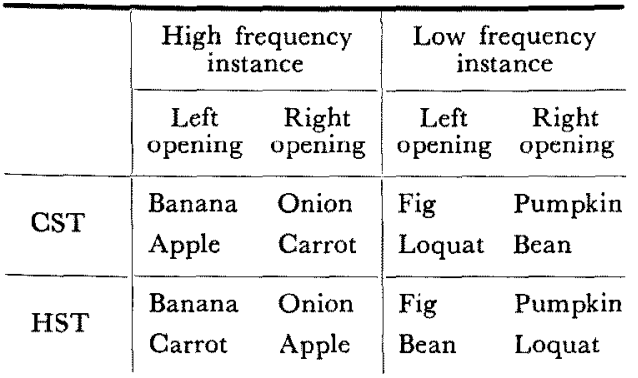

HST groups, respectively. Because of the skewness of the distributions in L-CST and L-HST groups, a nonparametric test was employed for statistical analysis. The results of median test showed that the performance of H-CST group ( $M d n=20.5)$ was significantly better at the $1 \%$ level with $\chi^{2}(1)=12.10$ than that of H-HST group $(M d n=34.0)$, and that no performance difference was found between L-CST $(M d n=53.0)$ and L-HST $(M d n=45.5)$ groups with $\chi^{2}(1)=0.42$.

\section{Discussion}

The obtained results in the present study showed that CST was learned faster than HST when $S$ s had high conceptual ability (Exp. I) and when Ss were given the sorting tasks with high-frequency instances (Exp. II). It is rather reasonable to conclude that these $S$ s have learned the sorting tasks by the use of concepts in a manner that an instance (e.g., banana) evoked its conceptual term (e.g., fruit) as a mediator which is connected to the particular sorting response. On the other hand, CST and HST were learned almost at the same rate when $S$ s had low conceptual ability (Exp. I) and when $S$ s were given the sorting tasks with low-frequency instances (Exp. II). These $S$ s may have learned the sorting tasks with little or no concept utilization in a manner that an instance is directly connected to the particular sorting response. It is evident from these findings that whether preschool children can learn in a mediational manner or not is dependent upon at least the $S$ 's ability relevant to the task in point and the nature of the learning task.

The obtained results in Experiment I are mainly due to the difference in $S$ 's conceptual ability because the same instances were employed for the $S \mathrm{~s}$ with higher and lower conceptual ability. As the performance difference between CST and HST would be responsible for the difference in possibility to use the conceptual terms, the $S$ s with higher conceptual ability learned faster CST than HST and the $S$ s with lower conceptual ability learned CST and HST almost at the same rate. In the present experiment $S$ 's conceptual ability was assessed by the summed scores of the two subtests. So it is not clear which one of these tests is more responsible for the concept utilization in the sorting tasks. Several studies (Johnson \& White, 1967; Sugimura, 1969; White \& Johnson, 1968) revealed that concept of dimensionality and abstraction ability are positively related to reversal learning: $S_{\mathrm{s}}$ with higher conceptual ability use mediation in reversal learning more than $S$ s with lower conceptual ability. These findings are in line with the present experiment.

The results obtained in Experiment II are interpreted to show that utilization of concept in learning the sorting tasks depends on the frequency of instances used in the tasks. Similar results were reported in the study of free recall: The category clustering tended to be greater for the highfrequency instances than for the low-frequency instances (Shin, 1972; Wachs \& Gruen, 1971). However, there still remain several points to be clarified in the future. (a) The high- and low-frequency instances used in this experiment were selected on the basis of the numbers of instances which were evoked from their conceptual terms. When learning the sorting tasks, on the other hand, $S$ s were given the individual instances and they 
had to evoke the conceptual terms from their instances by their own efforts. It is uncertain, at present, whether or not the high-frequency instances evoke their conceptual terms more readily than the lowfrequency ones do. (b) The superiority of CST over HST may be explained in terms of the formation of stimulus clusters suggested by Schaeffer and Ellis (1970). If the associative strengths of the two instances are stronger for CST (Banana and Apple) than for HST (Banana and Carrot), it would be predicted that the stimulus (instance) clusters in CST are formed more easily than those in HST, and that CST is learned faster than HST by the use of stimulus clusters. Relative effectiveness of the associative strengths among instances upon the sorting tasks will come into question. (c) The two sorting tasks with lowfrequency instances tended to be learned more slowly than those with high-frequency instances. This may be partially due to the difference in familiarity of the instances. It is probable that learning of unfamiliar instances is slower than that of familiar ones.

As is shown in Table 2, the overall performances in Experiment I were better than those in Experiment II. This may be partially due to the age difference of $S \mathrm{~s}$ used in these two experiments. Although the interactive effects of the two variables is not made clear in the present study, an inspection of Table 2 suggests that the concept utilization in the sorting tasks is more dependent upon $S$ 's conceptual ability rather than the frequency of instances. A number of studies have revealed developmental changes in the availability of verbal mediation. But it should still be noticed that even if the $S$ s are at the same level, some $S$ s are easy to use verbal mediation and others are not. More attention should be directed to variables other than age.

\section{REFERENCES}

HATA, Y. 1973 A study of the effect of verbal labelling in concept learning (II) Conceptual knowledge, abstraction ability and reversal. Japanese Journal of Educational Psychology, 21, 116-121. (In Japanese)

Johnson, P.J., \& White, R. M., Jr. 1967 Concept of dimensionality and reversal shift performance in children. Fournal of Experimental Child Psychology, 5, 223-227.

Kendler, H. H., Kendler, T.S., \& Marken, R.S. 1969 Developmental analysis of reversal and half-reversal shifts. Developmental Psychology, 1, 318-326.

Kendler, H. H., Kendler, T. S., \& SANders, J. 1967 Reversal and partial reversal shifts with verbal material. Journal of Verbal Learning and Verbal Behavior, 6, 117-127.

NEIMARK, E.D. 1974 Natural language concept: Additional evidence. Child Development, 45, 508-511.

Saltz, E., Soller, E., \& Siegel, I. E. 1972 The development of natural language concepts. Child Development, 43, 1191-1202.

Schaeffer, B., \& Ellis, S. 1970 The effects of overlearning on children's nonreversal and reversal learning using unrelated stimuli. Journal of Experimental Child Psychology, 10, 1-7.

Shin, K. 1972 Free recall in categorized lists (2). Proceedings in the 14th Meeting of the Japanese Association of Educational Psychology, 318-319. (In Japanese)

Sugimura, T. 1969 Reversal shift learning and abstraction ability in kindergarten children. Japanese Fournal of Educational Psychology, 17, 23-27. (In Japanese with an English summary)

Sugimura, T. 1974 A developmental change in learning of conceptual sorting and half-conceptual sorting tasks. Fapanese Journal of Psychology, 45, 249-255. (In Japanese with an English summary)

Sugimura, T., \& Ichikawa, H. 1975 Conceptual category norms for young children. Bulletin of Nara University of Education, 24, 1, 135146. (In Japanese with an English summary)

Wachs, T. D., \& Gruen, G. E. 1971 The effects of chronological age, trials, and list characteristics upon children's category clustering. Child Development, 42, 1217-1227.

White, R. M., Jr., \& Johnson, P.J. 1968 Concept of dimensionality and optional shift performance in nursery school children. Fournal of Experimental Child Psychology. 6, 113-119.

(Received July 25, 1975) 